\title{
Angiomyolipoma of the Liver: A Rare Benign Tumor Treated with a Laparoscopic Approach for the First Time
}

\author{
CHRISTOS DAMASKOS ${ }^{1,2^{*}}$, NIKOLAOS GARMPIS ${ }^{1 *}$, ANNA GARMPI ${ }^{3 *}$, AFRODITI NONNI ${ }^{4}$, \\ STRATIGOULA SAKELLARIOU ${ }^{4}$, GEORGIOS-ANTONIOS MARGONIS ${ }^{5}$, ELEFTHERIOS SPARTALIS ${ }^{2}$, \\ DIMITRIOS SCHIZAS ${ }^{6}$, NIKOLAOS ANDREATOS ${ }^{5}$, ELENI MAGKOUTI ${ }^{7}$, \\ ALEXANDROS GRIVAS $^{8}$, KONSTANTINOS KONTZOGLOU $^{1}$, MATTHEW J. WEISS $^{5}$ and EFSTATHIOS A. ANTONIOU ${ }^{1}$ \\ ${ }^{1}$ Second Department of Propedeutic Surgery, Laiko General Hospital, Medical School, \\ National and Kapodistrian University of Athens, Athens, Greece; \\ ${ }^{2}$ N.S. Christeas Laboratory of Experimental Surgery and Surgical Research, Medical School, \\ National and Kapodistrian University of Athens, Athens, Greece; \\ ${ }^{3}$ Internal Medicine Department, Laiko General Hospital, Medical School, \\ National and Kapodistrian University of Athens, Athens, Greece; \\ ${ }^{4}$ First Department of Pathology, School of Medicine, \\ National and Kapodistrian University of Athens, Athens, Greece; \\ ${ }^{5}$ Department of Surgery, The Johns Hopkins University School of Medicine, Baltimore, MD, U.S.A.; \\ ${ }^{6}$ First Department of Surgery, Laiko General Hospital, Medical School, \\ National and Kapodistrian University of Athens, Athens, Greece; \\ ${ }^{7}$ Department of Neurology, Agia Sofia General Children's Hospital, Athens, Greece; \\ ${ }^{8}$ Medical School, National and Kapodistrian University of Athens, Athens, Greece
}

\begin{abstract}
Background/Aim: Epithelioid angiomyolipoma of the liver is a rare benign mesenchymal tumor that usually presents in adult female patients. It most frequently occurs in the kidney, with the liver being the second most common site of involvement. Angiomyolipoma belongs to a family of tumors arising from perivascular epithelioid cells, but in rare cases may also have cystic features. We report our experience via the first case of hepatic angiomyolipoma treated by laparoscopic approach. Patients and Methods: We present the case of a 50-year-old female patient complaining of abdominal pain. Abdominal ultrasound (US) and Magnetic Resonance Imaging (MRI) revealed a $5 \times 3 \mathrm{~cm}$ mass located in the left liver lobe. The tumor was resected
\end{abstract}

This article is freely accessible online.

\footnotetext{
*These Authors contributed equally to this study.

Correspondence to: Dr. Nikolaos Garmpis, Second Department of Propedeutic Surgery, Laiko General Hospital, National and Kapodistrian University of Athens, Medical School; 17 Agiou Thoma Street, Athens, 11527, Greece. Tel: +30 6976291403, Fax: +302132061772, e-mail: nikosg22@hotmail.com
}

Key Words: Liver, angiomyolipoma, epithelioid, tumor, laparoscopic surgery. with a laparoscopic approach. Microscopic examination of the tumor revealed hepatic angiomyolipoma. Results: Twenty-seven months postoperatively, the patient remains fit and healthy. Conclusion: Angiomyolipoma can be removed by laparoscopy.

Epithelioid angiomyolipoma (AML) of the liver is a rare benign mesenchymal tumor that usually presents in adult female patients; nonetheless, several cases have also been reported among males. AMLs most frequently occur in the kidney, with the liver being the second most common site of involvement (1).

AMLs belong to a family of tumors arising from perivascular epithelioid cells (commonly called PEComas in the literature). Histological examination demonstrates that AMLs consist of adipose tissue, smooth muscle bundles, and thick-walled blood vessels in varying proportions, thus explaining the origin of their name.

Another characteristic feature of AMLs is the presence of epithelioid cells, particularly in the epithelioid variant of the tumor. Hepatic epithelioid AMLs may also have cystic features, but this is extremely rare (2).

In this report, we present the case of a 50-year-old female patient that was referred to our Department, after reporting discomfort in the right upper quadrant of the abdomen. Following a comprehensive initial work-up, a magnetic 

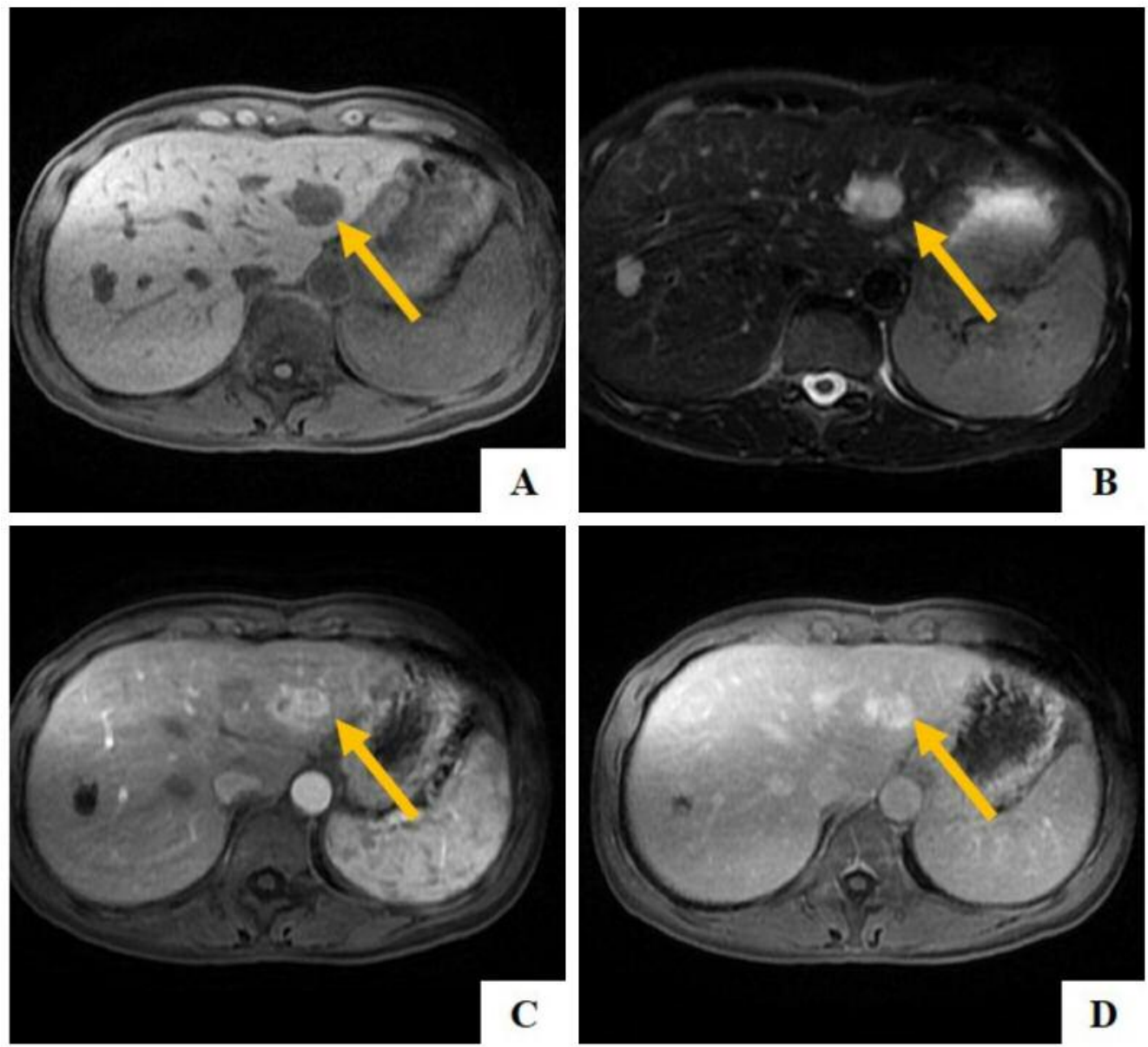

Figure 1. Angiomyolipoma located in the left liver lobe. A, B: On T1-weighted (A) imaging a sharply demarcated lesion with low signal intensity can be noted in the left liver lobe which shows a mildly hyperintense signal in T2-weighted imaging (B); $C, D$ : The lesion is markedly hypervascular in the arterial phase $(C)$ and remains hyperintense in the portalvenous phase $(D)$.

resonance imaging (MRI) scan demonstrated a well demarcated hepatic mass that was surgically resected. Pathologic examination revealed the presence of a hepatic epithelioid AML with extensive cystic degeneration, a rare but, fortunately, benign finding. Subsequently, the patient was advised to return for periodic follow-up. At the time of the last follow-up visit ( 27 months postoperatively), the patient remained asymptomatic with no evidence of recurrence.

\section{Case Presentation}

We present the case of a 50-year old female patient that was referred to our department, after complaining of right upper quadrant abdominal pain for the past 4 months. The pain was not related to food intake and failed to respond to painkillers. The patient's past medical history was unremarkable with no evidence of liver or biliary disease; furthermore, the patient reported no alcohol consumption. Clinical examination revealed tenderness in the right upper quadrant of the abdomen, as well as sensitivity to deep palpation in the right subcostal area. Laboratory examination demonstrated significant elevation of alanine aminotransferase (ALT) and aspartate aminotransferase (AST), as well as slightly decreased levels of total protein (Tp) and albumin (Alb). Alpha-fetoprotein (AFP), carcinoembryonic antigen (CEA) and carbohydrate antigen 199 (CA-19.9) levels were within normal limits. The patient underwent abdominal ultrasound (US) which revealed an isoechoic $5 \times 3 \mathrm{~cm}$ mass in the left liver lobe. Subsequently, a contrast enhanced MRI was performed; it demonstrated a welldefined cystic mass in the left liver lobe with a hypointense T1 signal and a hyperintense T2 signal (Figure 1A and B). A heterogeneous enhancement was observed in the walls of the cystic mass; furthermore, the presence of adipose tissue was demonstrated in the mass's cystic center (Figure 1C and D). 

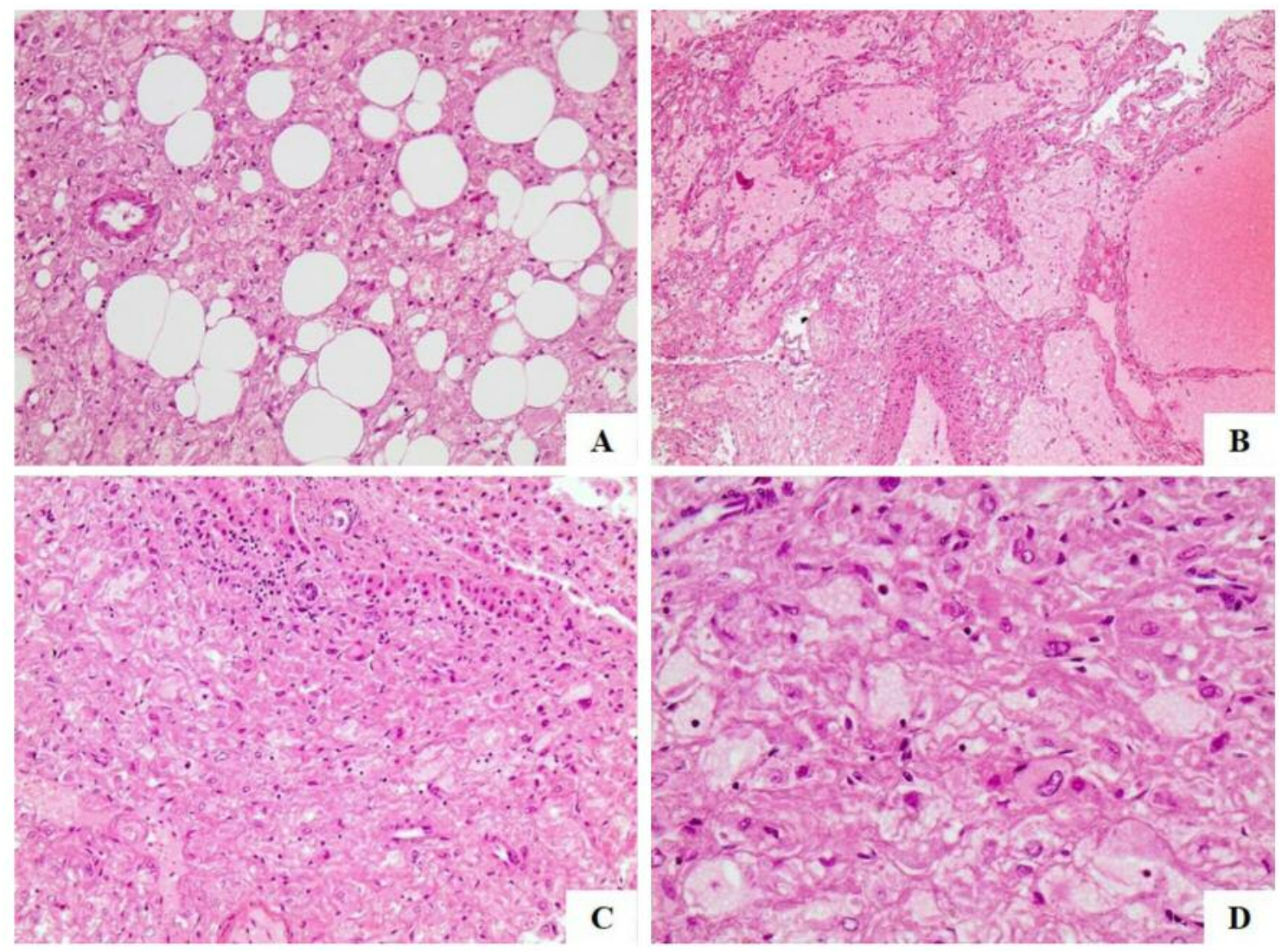

Figure 2. Histological examination of the resected hepatic angiomyolipoma. A: Neoplastic cells intermingling with scattered adipocytes $(H \& E, \times 200)$; $B:$ Neoplastic cells arranged around thick wound wall vessels and cystic spaces field with blood $(H \& E, \times 100) ; C$ : Neoplastic cells adjacent to normal liver (H\&C, ×200); D: Large Neoplastic cells with heosenophilic cytoplasm and enlarged nuclei with heosenophilic nucleoli $(H \& C, \times 400)$.

The patient underwent exploratory laparoscopy, which revealed a solid tumor in the left hepatic lobe. With the aid of intra-operative US, the tumor's borders and location with respect to the surrounding hepatic parenchyma and important vascular structures were carefully identified. No intrahepatic metastasis or satellite lesions were detected. Consequently, the decision was made to proceed with a left lateral hepatectomy (liver segments II and III) and concurrent cholecystectomy, both of which were performed laparoscopically. Following the successful completion of these procedures, a drainage tube was placed adjacent to the transected surface of the liver.

Gross examination revealed a circumscribed, smooth, multilobular tumor measuring $4 \times 4 \times 2.8 \mathrm{~cm}$. The cut surface of the tumor was spongiform and brownish in color, while the tumor wall demonstrated variable thickness. The tumor was resected intact and clear margins were achieved (R0 resection).

Microscopic examination of the tumor revealed multiple epithelioid cells with ample eosinophil cytoplasm, enlarged nucleus and prominent nucleoli. Interestingly, many of the epithelioid cells resembled ganglion cells; as noted above, the increased size of the nucleoli was their most remarkable feature. Neoplastic cells were arranged in broad solid sheets surrounding a prominent network of sinusoid-like vessels, filled with hemorrhagic fluid. Apart from epithelioid cells, the tumor also consisted of sinusoid-like vessels, spindle myoid cells and mature fat cells. Mitotic figures were not detected in any of these cellular populations, while focal areas of necrosis and hemorrhage were observed (Figure 2). A resection margin width of $3 \mathrm{~mm}$ was achieved.

Immunohistochemical analysis revealed that epithelioid tumor cells were positive for HMB45, Melan-A (MART1) (Figure 3A and B), as well as for smooth muscle actin (SMA) (Figure 3C). On the other hand, the spindle myoid cells were positive for SMA. Both epithelioid and spindle cells were negative for CD10, HePar-1 (Figure 3D), S-100, epithelial membrane antigen (EMA), AFP, c-kit, chromogranin A and synaptophysin. The proliferation index was very low in all examined cellular populations. 

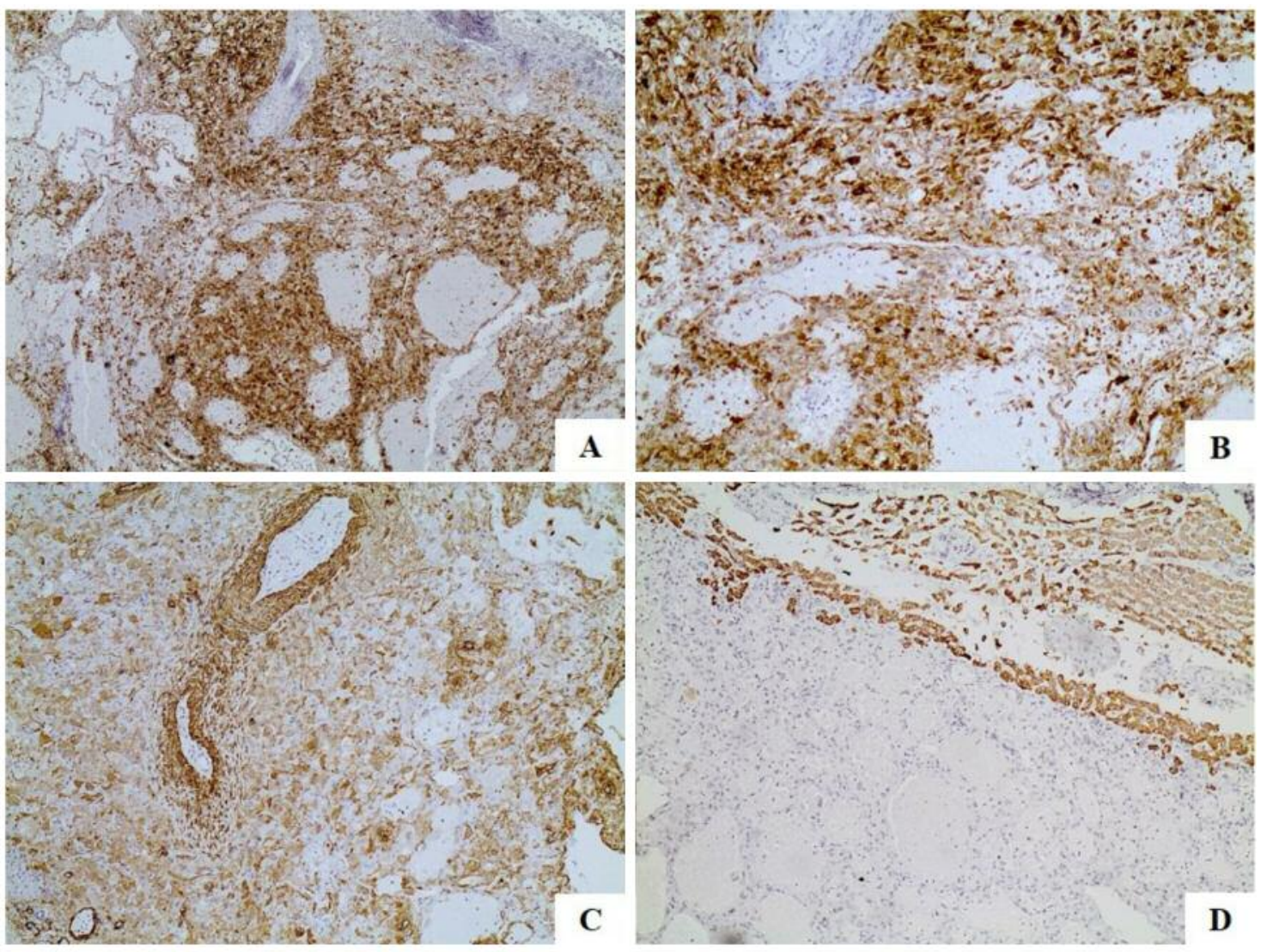

Figure 3. Immunohistochemical analysis of the resected hepatic angiomyolipoma. A: Positive reaction of neoplastic cells to MART1 ( $\times 100)$; B: Positive reaction of neoplastic cells to MART1 (×200); C: SMA expression of the neoplastic cells (×200); D: The neoplastic cells are negative to HeParl ( $\times 200)$.

The patient's post-operative course was uneventful and all routine laboratory tests were within normal limits. The drainage tube was removed after the 4th post-operative day and the patient was discharged 8 days later. On follow-up examination, the patient remained asymptomatic and healthy to the present day. Computed tomography scans were also performed 6, 12, 18 and 24 months postoperatively; both imaging studies demonstrated no evidence of recurrence or other abnormal findings. A total of 27 months postoperatively, the patient remains fit and healthy.

\section{Discussion}

AMLs are mesenchymal tumors of benign nature, most commonly encountered in the kidney and closely associated with tuberous sclerosis (3). The liver is the second most common site of AML occurrence (1). Hepatic AML can present similarly to malignant tumors, such as hepatocellular carcinoma, both clinically and radiologically. Hepatic AMLs are difficult to diagnose radiologically, because of the variability of their growth patterns and their proximity to major hepatic vascular structures. However, the presence of mature adipose tissue may facilitate the accurate preoperative diagnosis of this rare clinical entity (4). It has been reported that hepatic AMLs are more commonly located in the right lobe (5). However, solitary involvement of the left lobe is also possible, as illustrated by the present case.

Hepatic AML was first described in 1976 by Ishak et al. (6) and is known to present with a wide variety of growth patterns. These variations reflect the distribution and degree of differentiation of myoid cells within the tumor and have been described by Tsui et al. (7). Several distinct AML histologic types have been identified, including the lipomatous, myomatous, angiomatous, trabecular, pelioid, inflammatory and mixed variants (8). Gross examination of hepatic AMLs usually reveals soft, solitary, well encapsulated tumors (4). The presence of cystic elements filled with hemorrhagic contents, as in the present case, is 
very rare. Regardless of their site of occurrence, AMLs are generally composed of epithelioid and spindle myoid cells, mature adipose tissue and sinusoid-like vessels. The presence of multiple epithelioid cells within a tumor can be misleading and may render the differentiation of AML from hepatocellular carcinoma challenging (9).

In this report, we presented a rare case of hepatic epithelioid AML with extensive cystic degeneration in a 50year-old woman. The tumor demonstrated the characteristic pathologic features of AML, such as the presence of spindle myoid cells, mature adipose tissue and sinusoid-like vessels; epithelioid cells were the predominant cell type. Immunohistochemisrty was pathognomonic for AML, as epithelioid and spindle myoid cells were positive for melanocytic (HMB45) and myogenic (SMA) markers, but negative for epithelial markers (EMA).

The treatment of hepatic AML is mostly surgical. It is recommended that small lesions should be closely observed and treated conservatively, while larger lesions should be excised with clear margins. The present report is, to our knowledge, the first to describe the laparoscopic excision of a hepatic AML; a more detailed study of the efficacy of laparoscopic techniques in this setting should be considered.

\section{Conclusion}

AML is a rare, benign mesenchymal liver tumor that may present similarly to a hepatic malignancy. We herein presented a rare case of hepatic AML that was uncharacteristically located in the left liver lode and demonstrated cystic degeneration with limited intracystic hemorrhage. AML should always be a part of the differential diagnosis in patients that present with solitary hepatic lesions; familiarity with the characteristic features of this uncommon clinical entity is essential to formulating an appropriate diagnostic and therapeutic strategy.

\section{Acknowledgements}

The Authors would like to thank Dr. Zormpala A. for performing imaging examinations.

\section{References}

1 Alatassi $\mathrm{H}$ and Sahoo S: Epithelioid angiomyolipoma of the liver with striking giant cell component: fine-needle aspiration biopsy findings of a rare neoplasm. Diagn Cytopathol 37: 192-194, 2009.

2 Zhou Y, Chen F, Jiang W, Meng Q and Wang F: Hepatic epithelioid angiomyolipoma with an unusual pathologic appearance: expanding the morphologic spectrum. Int J Clin Exp Pathol 7: 6364-6369, 2014.

3 Rakowski SK, Winterkorn EB, Paul E, Steele DJ, Halpern EF and Thiele EA: Renal manifestations of tuberous sclerosis complex: Incidence, prognosis, and predictive factors. Kidney Int 70: 1777-1782, 2006.

4 Bakhotmah MA and Yamasaki S: Hepatic angiomyolipoma. HPB Surg 8: 133-137, 1994.

5 Ishak KG: Mesenchymal tumors of the liver. In: Hepatocellular Carcinoma. Okuda K and Peters RL (eds.). New York, John Wiley \& Sons, pp. 247-307, 1976.

6 Tsui WM, Colombari R, Portmann BC, Bonetti F, Thung SN, Ferrell LD, Nakanuma Y, Snover DC, Bioulac-Sage P and Dhillon AP: Hepatic angiomyolipoma: a clinicopathologic study of 30 cases and delineation of unusual morphologic variants. Am J Surg Pathol 23: 34-48, 1999.

7 Shi H, Cao D, Wei L, Sun L and Guo A: Inflammatory angiomyolipomas of the liver: a clinicopathologic and immunohistochemical analysis of 5 cases. Ann Diagn Pathol 14: 240-246, 2010.

8 Talati H, Radhi J, Popovich S and Marcaccio M: Hepatic Epithelioid Angiomyolipoma: Case Series. Gastroenterology Res 3: 293-295, 2010.

9 Romano F, Franciosi C, Bovo G, Cesana GC, Isella G, Colombo $\mathrm{G}$ and Uggeri F: Case report of a hepatic angiomyolipoma. Tumori 90: 139-143, 2004.
Received August 25, 2017

Revised September 14, 2017

Accepted September 19, 2017 